\title{
Fertile ground
}

\author{
Stephen B Hanauer
}

As I peruse the general medical literature for inspiration when writing these monthly editorials, I often find complementary articles that allow me to expound on topics that might not have immediate or apparent synergy. Inspiration struck this month when, immediately after reading an editorial by lain Chalmers on "Why fair tests are needed: a brief history" (ACP J Club [2006] Jul-Aug; 145: A8-A9), I came across an article by Steures et al. titled "Intrauterine insemination with controlled ovarian hyperstimulation versus expectant management for couples with unexplained subfertility and an intermediate prognosis: a randomised clinical trial" (Lancet [2006] 368: 216-221).

Chalmers provided a brief adaptation of information from the James Lind Library (www. jameslindlibrary.org) that highlighted several examples of how health professionals have, inadvertently, harmed their patients "... because treatment decisions were not informed by what we now consider to be reliable evidence about the effects of treatment." Chalmers reviewed the historic use of bloodletting, as well as more recent, inappropriate, advocations-placing infants in a prone position to sleep (now recognized to increase the risk of SIDS), the use of class I antiarrythmic drugs after acute myocardial infarction (which actually increases the risk of sudden death), and the use of diethylstilbestrol to prevent miscarriage (now known to increase the rate of clear cell adenocarcinoma of the vagina and cervix in female children). Other recent examples of the use of unreliable evidence to guide treatment include hormone replacement therapy to reduce heart disease in postmenopausal women, supplementation with vitamin $\mathrm{C}$ to prevent colds, or supplementation with vitamin $\mathrm{A}$ or $\mathrm{E}$ to prevent heart disease or cancer.

The report of the multicenter clinical trial by Steures and colleagues could debunk another
Fair tests, controlled trials and the principles of evidence-

based medicine are crucial assuming we ask the right questions

SB Hanauer is Editorin-Chief of Nature Clinical Practice Gastroenterology \& Hepatology.

\section{Competing interests}

The author declared he has no competing interests.

www.nature.com/clinicalpractice doi:10.1038/ncpgasthep0612 'potentially beneficial' practice in couples with unexplained subfertility. Approximately $10 \%$ of couples who want to have children fail to conceive within a year of trying, and there are a substantial proportion of such couples (up to $20-30 \%$ ) for whom the cause of their infertility is not identified, and for whom intrauterine insemination with ovarian hyperstimulation $(\mathrm{IIOH})$ has been proposed. These investigators randomly allocated 253 couples from multiple centers in the Netherlands who were predicted to have a $30-40 \%$ probability of a spontaneous ongoing pregnancy within 12 months to receive $\mathrm{IOH}$ or expectant management for 6 months. After 6 months, $33 \%$ of the women in the $\mathrm{IIOH}$ group and $32 \%$ in the expectant management group became pregnant. Interestingly, the miscarriage rate was greater in the $\mathrm{IOH}$ group (31\%) than the expectant group (15\%), which led to a greater number of ongoing pregnancies in the expectant management group than the intervention group ( $27 \%$ vs $23 \%$, relative risk 0.85 , 95\% Cl 0.63-1.1). The relative risk for a live birth also favored the expectant management group (relative risk 0.86, 95\% Cl 0.54-1.4). Essentially, there were no significant differences between the groups, and it was notable that in the 127 couples randomly allocated to $\mathrm{IOH}$ there were six spontaneous pregnancies before $\mathrm{IOH}$ was initiated and seven spontaneous pregnancies between cycles of $\mathrm{IIOH}$ or in vitro stimulation. I was also surprised to learn that the ongoing pregnancy rate was only $4 \%$ (expected $9 \%$ ) per cycle in the $\mathrm{IIOH}$ group.

A favorite quote of mine (author unknown) is that "Those with enthusiasm have no controls; those with controls have no enthusiasm." Fair tests, controlled trials and the principles of evidence-based medicine are crucialassuming we ask the right questions. 